\title{
Cervical screening: why young women should be encouraged to be screened
}

\author{
Amanda Herbert, Gillian Holdsworth, Ali A Kubba
}

\begin{abstract}
Background The English National Health Service Cervical Screening Programme (NHSCSP) recommendation not to offer cervical screening to women aged 20-24 years is considered in the context of national rates of cervical intraepithelial neoplasia grade 3 (CIN3) and invasive cervical carcinoma, falling screening coverage in young women, detection of screen-detected invasive cancers and risks of excisional treatment of CIN.
\end{abstract}

Methods Registrations of invasive and in situ cervical carcinoma were obtained from the Office for National Statistics, data on screening coverage and cytology results from the NHSCSP website and data on screendetected cancers from an audit at Guy's \& St Thomas' NHS Foundation Trust (GSTFT).

Results Before and after the introduction of organised screening in England, CIN3 was primarily detected in women aged 20-39 years. Increasing rates of CIN3 were recorded in women aged 20-24 years during the last decade (3000-4000 cases per year) despite falling screening coverage. The peak incidence of invasive cancer in screening age groups is now 35-39 years. At GSTFT in 1999-2006, 24 of 32 cancers (75\%) in women aged 20-34 years were screen-detected and that percentage declined in subsequent 15-year age bands $(p \leq 0.0001)$.

Discussion and conclusions Delaying the age for screening eligibility carries a risk of CIN becoming more extensive, and therefore more difficult to excise, as well as a risk of progression. The NHSCSP should reconsider its decision and encourage young women to be screened, not excluding those aged 20-24 years. Facilities for taking the tests should be made more convenient. Women should be informed that low-grade CIN is potentially reversible and may safely be monitored. Cervical screening also provides an opportunity for education on healthy lifestyles and safer sex while treatment should be reserved for high-grade CIN.

Keywords cervical screening, cervical intraepithelial neoplasia, cervical carcinoma incidence, CIN, microinvasive carcinoma, screen-detected cancer

J Fam Plann Reprod Health Care 2008; 34(1): 21-25

(Accepted 18 November 2007)

\section{Introduction}

Invasive cervical cancer incidence and mortality have fallen by nearly $50 \%$ since screening was centrally organised in 1988, despite an escalating risk of disease seen across Europe in birth cohorts of women born since the 1940s. ${ }^{1}$ Furthermore, birth cohort trends in mortality in England and Wales declined steeply after 1988 in all screening age groups, including women under 35 years whose mortality rates at the time were among the highest in the world. ${ }^{2}$ That reversal in trend, which persisted in subsequent decades of life, suggests that an epidemic of cervical cancer was avoided by screening women when they were young: an epidemic likely to reflect the greater sexual freedom allowed by the availability of reliable contraception since the late 1960 s. $^{2}$

In 2004, the National Health Service Cervical Screening Programme (NHSCSP) introduced new recommendations for screening intervals. ${ }^{3}$ Women under 25 years would no longer be offered screening and the intervals would be standardised as 3-yearly for women aged 25-49 years and 5-yearly for those aged 50-64 years. A case-control study had shown that invasive cancer was rare in women under 25 years and that screening was

Guy's \& St Thomas' NHS Foundation Trust, London, UK

Amanda Herbert, MBBS, FRCPath, Consultant

Southwark Primary Care Trust, London, UK

Gillian Holdsworth, MB BCh, FFPH, Consultant

Lambeth Primary Care Trust and Guy's \& St Thomas' NHS

Foundation Trust, London, UK

Ali A Kubba, FRCOG, FFFP, Consultant

Correspondence to: Dr Amanda Herbert, Histopathology Department, Second Floor, North Wing, St Thomas' Hospital, London SE1 7EH, UK. E-mail: amanda.herbert@kcl.ac.uk

\section{Key message points}

- Most cervical intraepithelial neoplasia grade 3 (CIN3) is detected in women aged 20-39 years, and appropriate treatment has reduced the incidence of invasive cervica cancer despite increasing rates of pre-invasive disease.

- Rates of CIN3 have increased in women aged 20-24 years despite falling screening coverage, and the NHS Cervical Screening Programme (NHSCSP) recommendation not to screen these women may mean that a few will enter the programme 'too late' to avert cervical cancer.

- Most invasive cancers in women aged under $\mathbf{4 0}$ years are screen-detected at an early and treatable stage, when fertility may be preserved, and the peak incidence in age groups eligible for screening is 35-39 years.

- Giving accurate information about low-grade CIN, together with promoting healthy lifestyles and safer sex, empowers young women to look after their sexual health.

relatively ineffective in preventing fully invasive cancer (microinvasive cancers were excluded from the analysis) in younger compared with older women, justifying a shorter interval in the former and longer in the latter. ${ }^{4}$ Screening women aged under 25 years was considered to cause more harm than good, a view that was supported by the International Agency for Research on Cancer. ${ }^{5}$

Concerns have been raised about the safety of the new starting age for cervical screening. $2,6-8$ We are also concerned that stating that screening causes more harm than good in women aged 20-24 years might give the wrong message to young women in general, among whom local (and national) coverage rates are falling. 9 A study in Iceland has shown a benefit in reducing the age of the first invitation to include women below 25 years. ${ }^{10}$ In order to investigate this, we analysed data from an audit of cervical cancer in our 
area, which encompasses a relatively young population with a high prevalence of sexually transmitted infections (STIs). We believe that levels of high-grade cervical intraepithelial neoplasia (CIN) - particularly CIN3 - and screen-detected cancers - particularly microinvasive cancers - should be considered as well as overall invasive cancer rates if the true effectiveness of screening is to be examined. We therefore examined national rates of carcinoma in situ and invasive cervical carcinoma alongside NHSCSP data for cytology reporting rates and screening coverage. In the absence of national or regional data on screen-detected cancers or microinvasive carcinomas, which are not identified as such among national registrations of invasive cancers, we present local cervical cancer audit data from Guy's \& St Thomas' NHS Foundation Trust (GSTFT). This information will be available in the future at a regional and national level now that invasive cancer audit data are being collected and analysed centrally. ${ }^{11}$

\section{Data collection and analysis}

Registrations and rates per 100000 total female population of invasive and in situ cervical carcinoma are published annually by the Office for National Statistics (ONS) and data since 1992 are available online. ${ }^{12}$ Complete ExcelTM spreadsheet tables for 1971-2004 were requested and received by email from ONS early in 2007. Registrations since 1995 are for England alone and prior to that date included Wales. Figures for 2005 will not be available until the end of 2007. Registrations are recorded in 5-year age bands for each year, which allows data points at 5-year intervals to be analysed for birth cohorts. Registrations are recorded as total numbers, as rates per 100000 women in each 5-year age band and as rates for the total female population each year. Registrations of CIN3 are included with in situ carcinoma since 1984, are based on histological diagnoses and include adenocarcinoma in situ. Rates (described as 'incidence' by ONS) of CIN3 are based on registrations per 100000 total population and depend on screening coverage, only relating to prevalence of disease when coverage is both high and constant. Data on cervical screening coverage and cytology reports were taken from the Statistical Bulletin (England) 2005-06.9

Local data on cervical cancers diagnosed between 1999 and 2006 were taken from audits of invasive cervical cancer that have been carried out in our Trust since January 1999, using a template now used by the London Quality Assurance Reference Centre (QARC). Screen-detected cancers are defined as those detected through the investigation of abnormal cytology in asymptomatic women. Cases since 2000 have already been submitted to the London QARC and will be included in the regional and national audit of screening histories that is currently underway.

\section{Statistical analysis}

Chi-square for trend was calculated using the StatCal ${ }^{\circledR}$ program in the EPI6 software developed by the World Health Organization, Geneva, Switzerland and the Centers for Disease Control, Atlanta, GA, USA.

\section{Results}

\section{Incidence of invasive cancer and CIN3 in England}

Before the introduction of organised screening, the incidence of invasive cervical carcinoma increased substantially in women aged 30-34 and 35-39 years compared with the same age bands 15 years earlier and reached a high point in about 1989 (Figure 1). Since then incidence has decreased by more than $50 \%$ in all age bands over the age of 30 years. Notably, incidence in the high-risk

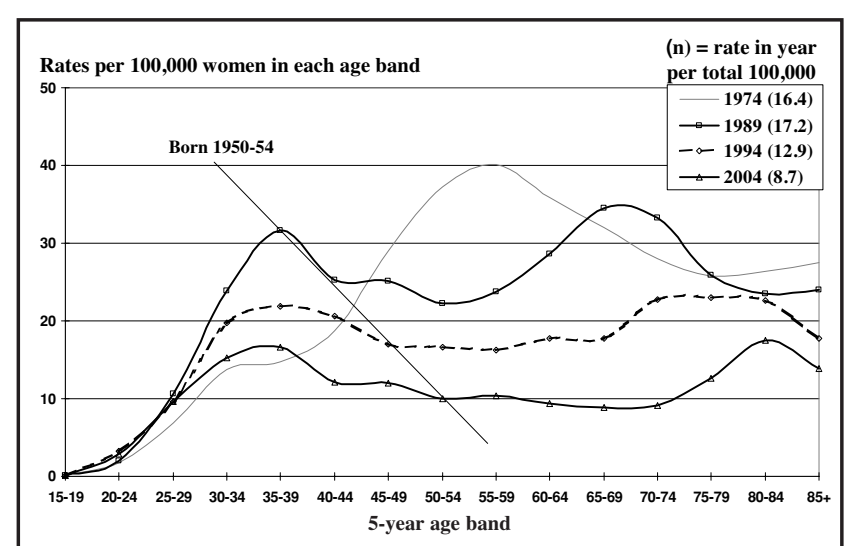

Figure 1 Incidence of invasive cervical carcinoma, England and Wales 1974 and 1989; England 1994 and 2004. Source: Office for National Statistics 12

cohort of women born in 1950-1954, who were aged 35-39 years in 1989, decreased in the following 15 years from 31.6 to $10.0(68.4 \%)$ during a period of life when it would be expected to increase (Figure 1). Although invasive cancer rates in women aged 20-24 and 25-29 years are low, they have not fallen and remain higher than in 1974.

Overall rates of CIN3 (registered as carcinoma in situ) have been relatively constant since 1989 and far higher than in 1974 (Figure 2). Rates have been consistently higher in women in their 20 s and 30 s compared with later decades of life. Since 1989, rates have increased in women aged 20-24 and 25-29 years, while decreasing in those aged 35-39 years and above; the increase in younger women has taken place against a background of falling screening coverage since 1995 (NHSCSP Statistical Bulletin 2005/2006), ${ }^{9}$ which has been most pronounced in women aged 20-24 years (Figure 3). Registrations of CIN3 in women aged 20-24 years represent an increasing percentage of total registrations: $2276 / 18441(12.3 \%)$ in $1989,3103 / 19817(15.7 \%)$ in $1994,3539 / 22346(15.8 \%)$ in 1999 and $3467 / 17935(19.3 \%)$ in 2004 . This trend is highly significant $(p \leq 0.00001)$.

The rapid fall in incidence of invasive carcinoma, especially in the 5 years between 1989 and 1994, followed a surge in rates of CIN3 registered during the late 1980s, which involved relatively more women aged over 40 years than before or since. Invasive carcinoma has largely been controlled in women aged 40-44 years and above, leaving the peak at age 35-39 years (Figure 1).

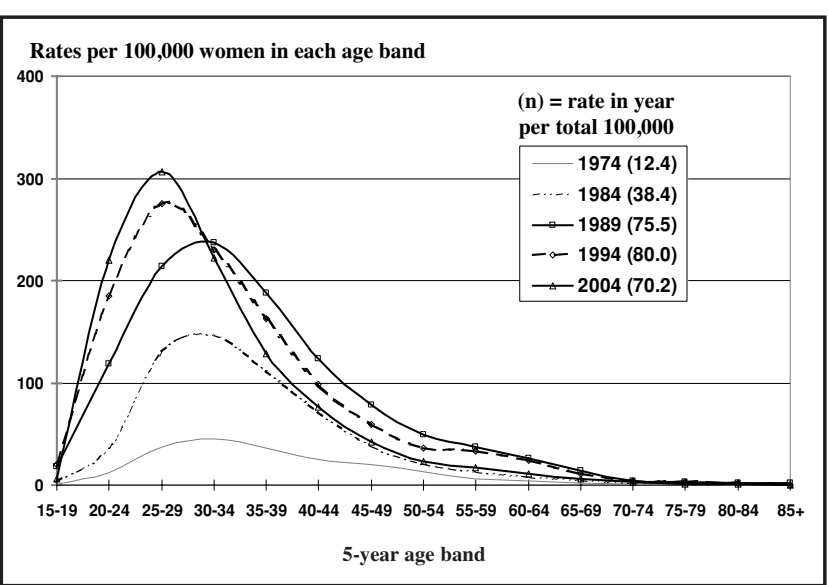

Figure 2 Incidence of carcinoma in situ of the cervix; England and Wales 1974, 1984 and 1989; England 1994 and 2004. Source: Office for National Statistics ${ }^{12}$ 


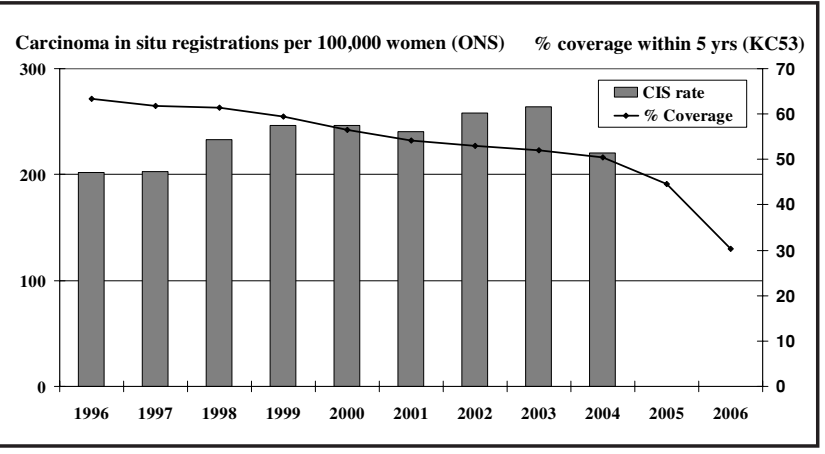

Figure 3 Carcinoma in situ rates and screening coverage, age 20-24 years. Source: Office for National Statistics ${ }^{12}$ and Statistical Bulletin (England) 2005-069

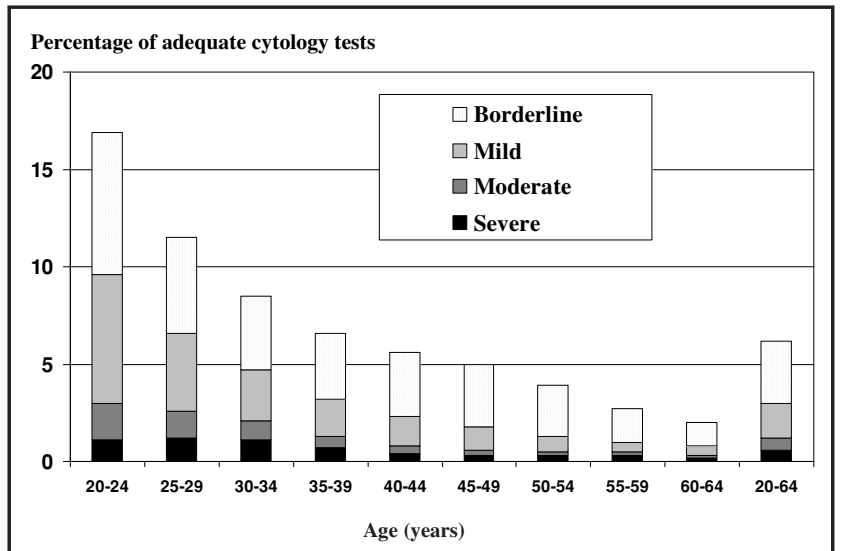

Figure 4 Cytological abnormalities, England 2005-2006. Source: Statistical Bulletin (England) 2005-069

\section{Rates of abnormal cervical cytology in England}

Rates of moderate and severe dyskaryosis on cytology in England (recorded in the NHSCSP Statistical Bulletin) ${ }^{3}$ are similar in women aged 20-24 and 25-29 years and decline in women aged 40 years and over (Figure 4), demonstrating a similar age profile to the ONS data for CIN3 (Figure 2). There is a high prevalence of low-grade cytology (mild dyskaryosis and borderline change) in young women, particularly those aged 20-24 years (Figure 4).

\section{Invasive cervical cancer at GSTFT}

Data on 108 cases of invasive cervical cancer diagnosed between 1999 and 2006 at GSTFT are presented in Figure 5. There were two cancers in women aged 20-24 years, 12 in women aged 25-29 years and 18 in women aged 30-34 years. Fifty-eight of 59 screen-detected cancers

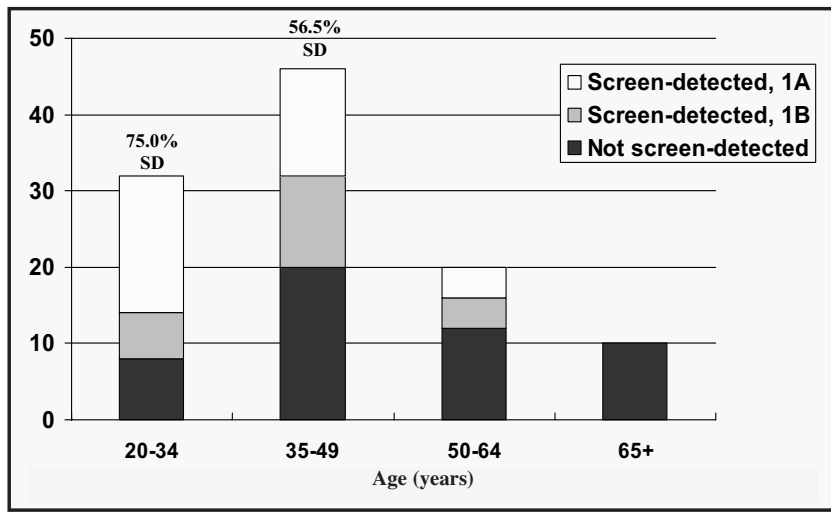

Figure 5 Data on 108 invasive cervical cancers (58 screendetected and 50 symptomatic cancers) diagnosed between 1999 and 2006 at Guy's \& St Thomas' NHS Foundation Trust (GSTFT) were International Federation of Gynecology and Obstetrics (FIGO) Stage 1 and 36/58 (62.1\%) were Stage 1A. Eighteen of $36(50 \%)$ Stage 1 A cancers were in women aged 20-34 years. Screen-detected cancers represented $75 \%$ of cancers in women aged $20-34$ years, $56.5 \%$ aged $35-49,40 \%$ aged $50-64$ and $0 \%$ aged 65 years and over, which was a significant trend $(p \leq 0.0001)$. The small number of cases in women aged 65 years and over is explained by the relatively young population in Lambeth and Southwark where women over 65 years represent $12.3 \%$ of the population compared with $22.5 \%$ in England as a whole (Source: local Primary Care Trust data).

\section{Discussion}

The age and birth cohort distributions of invasive cervical carcinoma incidence in England demonstrate the striking 'period-specific' effect of organised screening described by Bray et al. ${ }^{1}$ and the increased trend of 'cohort-specific' risk in women born since $1940^{1}$ supporting the mortality trend conclusions of Peto et al. ${ }^{2}$ Incidence in women aged 40 years and over in 2004 approaches that of low-risk cohorts born in the 1930s. The peak incidence of invasive cancer is now in women aged 35-39 years. Risk in birth cohorts later than 1960 is impossible to assess from incidence alone because a sharp decrease in incidence since 1989 is seen in women as young as 30-34 years of age.

Trends in CIN3 have not previously been considered in the context of effectiveness of screening but may be used as a surrogate for cancer risk $^{13}$ as long as levels of coverage are taken into account. There has been a marked increase of CIN3 in women aged 20-24 and 25-29 years since 1989 , despite falling screening coverage in those age bands since 1995, while rates have decreased in older age bands (Figure 2). Thus, there is strong evidence that the underlying risk of development of cervical cancer has increased in recent birth cohorts as would be expected from increasing rates of STIs and earlier age at first intercourse. ${ }^{14}$ The latest figures for 2006-2007 have recently been published and show that coverage has fallen further in the last 10 years from $61.8 \%$ to $24.0 \%$ in women aged 20-24 years (as expected) and from $78.2 \%$ to $68.2 \%$ in women aged 25-29 years. ${ }^{15} \mathrm{We}$ believe that the screening policy needs to take account of opposing trends of increasing risk and falling cervical screening coverage in young women. Unless screening coverage is improved, the incidence of cancer in women in their 30 s could increase as it did in the 1980s.

During the last decade of ONS records, 3000-4000 cases of CIN3 were registered each year in women aged 20-24 years representing a significantly increasing percentage of total CIN3 diagnoses. If not detected by screening, some of those lesions would have remained non-invasive for many years and could have been detected at a subsequent round of screening; some would have regressed, which would be much less likely with CIN3 than CIN2; but some would undoubtedly have progressed and would have been at risk of becoming invasive before the next round of screening; or of becoming more extensive, and therefore more difficult to excise completely. CIN3 first spreads by lateral extension, with involvement of endocervical crypts, and is usually widespread by the time invasive cancer develops. ${ }^{16}$ Thus women with microinvasive cancer usually require more extensive treatment than is needed for CIN3 and CIN2. Knife cone biopsy is often recommended for microinvasive carcinoma even if CIN3 appears to be completely excised on large loop excision of transformation zone (LLETZ). ${ }^{17,18}$

CIN represents a spectrum between reversible human 
papillomavirus (HPV) infection and CIN3. Progression is more likely and regression less likely the higher the grade of CIN. A meta-analysis of follow-up studies indicated that $11 \%$ of CIN1 and $22 \%$ of CIN2 progress to CIN $3 .{ }^{19}$ Boyes et al. estimated from modelling that $26 \%$ and $53 \%$ of CIN3 would progress to invasion in younger and older women, respectively. ${ }^{20}$ Although CIN3 may regress without treatment, in clinical practice there is no medicolegal justification for its follow-up rather than treatment ${ }^{21}$ as shown by the report of the Cartwright enquiry that followed. ${ }^{22}$ The risk of leaving high-grade CIN undetected and therefore untreated is demonstrated in practice by observations of the histories of women with invasive cervical cancer who have previously been screened. Review of all aspects of the screening history often reveals inadequate sampling of the cervix, undercalling of high-grade cytology, delays in referral, incomplete treatment of high-grade CIN and failures of follow-up. ${ }^{23-25}$

Sasieni et al. observed that cancers in women who had previously been screened (interval cancers) were more often seen in younger age groups. ${ }^{4}$ Sykes et al. showed that $65 \%$ of 29 women with microinvasive carcinoma and $85 \%$ of 56 controls with CIN3 had been screened prior to the index smear that resulted in diagnosis. ${ }^{26} \mathrm{~A}$ study in Southampton led by one of the authors (AH) also showed that CIN3 and cervical glandular intraepithelial neoplasia (CGIN) were seldom picked up by the first cervical smear test and most women with those diagnoses had previous negative or mildly abnormal cytology before the test that recommended investigation. ${ }^{27}$ In that study only $20 \%$ of CIN3 and CGIN were detected on the first test and the percentage was lower in women under rather than over the age of 40 years. ${ }^{27} \mathrm{~A}$ single cytology test is known to be less sensitive than a series of tests in the detection of prevalent high-grade CIN and cancer ${ }^{28}$ and neither CIN3 nor microinvasive carcinoma is likely to be detected on the first test.

Organised screening has had little effect on the incidence of invasive cervical cancer in women under the age of 30 years (Figure 1) and Sasieni et al. concluded from the case-control study that screening was less effective in younger compared with older women. ${ }^{4}$ Even if not prevented, some of those cancers may have been screen-detected in asymptomatic women. The casecontrol study of Sasieni et al. did not record how many fully invasive cancers were screen-detected. 4 The GSTFT audit showed that $75 \%$ of cancers in women aged 20-34 years were screen-detected and that percentage declined significantly with age. Although more than half the screen-detected cancers were microinvasive, 42/58 $(47.9 \%)$ were Stage $1 \mathrm{~B}$. There are very few reports in the literature describing screen-detected cervical cancers but a 12-year study of 382 cancers showed a similar finding by the time that successful screening was in place. ${ }^{29}$ Between 1985-1987 and 1994-1996, although the incidence of invasive cancer fell in line with England as a whole, there was no fall in the small number of cancers in women aged 20-34 years. Over that time period and in that age group there was a significant trend $(p=0.015)$ from symptomatic to screen-detected cancers: 4/14 $(40 \%)$ in 1985-1987 were screen-detected and 9/12 (75\%) in 1994-1996 (data calculated from Table 3). ${ }^{29}$ All but one of the screen-detected cancers in the GSTFT series (Figure 5) and $90.4 \%$ of those in the Southampton series were Stage 1 but less than half of the latter were Stage 1A. Pretorius et al. reported that $87 \%$ of cancers detected in women presenting with abnormal cervical smears were Stage 1 compared with $40 \%$ of those with symptomatic cancers. ${ }^{30}$ Although screen-detected cancers were not defined as such, Sigurdsson et al. reported that younger women had a significantly better prognosis than older women, which was attributed to their having previously attended screening and having earlier stage cancers. ${ }^{31}$ Survival is related to stage and fertility is usually preserved in women treated for Stage $1 \mathrm{~A}$ tumours and some for Stage 1B. ${ }^{32}$ Screen-detected cancers include early adenocarcinomas, ${ }^{29}$ which are less likely to be prevented by screening than squamous cell carcinomas. ${ }^{33}$

The Icelandic programme showed the benefit, particularly in terms of detection of microinvasive cancers in young women, of bringing forward the first invitation from age 25 to 20 years. ${ }^{10}$ In the national casecontrol study discussed above, 537/2753 total cancers in the series were Stage 1A and of those $54.2 \%$ were in women aged $25-39$ years. ${ }^{4}$ In the GSTFT study, $50 \%$ of Stage $1 \mathrm{~A}$ cancers were in women aged 20-34 years. Treatment of microinvasive cancer aims to eradicate cancer and save life, and is often more aggressive than treatment for CIN2 or CIN3. Thus, detecting early invasive cancers is a qualified advantage of screening asymptomatic younger women which, although it reduces mortality, does not reduce incidence and its treatment tends to incur greater morbidity compared with CIN3.

The disadvantages of screening women aged 20-24 years include the high prevalence of low-grade abnormalities (mostly representing transient HPV infection) in that age group, the associated risk of psychological trauma and the risk of over-zealous treatment of potentially reversible lesions. These disadvantages, together with the rarity of invasive carcinoma in that age group, are among the reasons cited by the NHSCSP for the new restrictive recommendation. Furthermore, excisional treatment of CIN has recently been associated with an increased risk of preterm delivery, ${ }^{34}$ which was not shown with ablative techniques. ${ }^{35}$ The risk increases with the depth of tissue removed from the cervix, ${ }^{36}$ which is more likely to be necessary with more advanced lesions. Decisions about treatment, to which women themselves should contribute, should depend on a balance between the risk of progression to more extensive CIN or cancer, the likelihood of regression and the risks of treatment.

We recommend that treatment of low-grade CIN should be avoided, especially in young women, but that cytological or colposcopic follow-up should be recommended to make sure that the changes do not persist or progress. Counselling should emphasise the potential reversibility of these lesions and should include advice on changes in lifestyle, such as cessation of smoking, improvement of diet and use of condoms, all of which may increase the likelihood of natural regression. ${ }^{37-39}$

Low perception of risk in the general and professional population, limited access to general practitioner (GP) clinics outside working hours and the withdrawal of cytology services at some sexual and reproductive health (SRH) clinics may have all contributed to the decline in screening coverage. In the past, SRH clinics have provided a flexible service in terms of clinic times without the need for appointments but are now reluctant to provide services for which GPs rather than providers are paid on the basis of coverage targets. Positive steps should be taken to fund community clinics to carry out cervical screening at convenient times as part of a sexual health package of care. 
Women might be more likely to accept invitations for screening if reassured that monitoring low-grade abnormalities is safe and that identifying and treating highgrade CIN, which is increasingly diagnosed in young women, prevents development of more extensive disease as well as cancer. But women will only accept screening invitations if the facilities for having their 'smears' taken are more accessible. Women aged 20-24 years should no longer be given the message that screening causes more harm than good and should not be actively discouraged from being screened.

\section{Acknowledgements}

The authors would like to thank Margaret Sheldrake of the Office for National Statistics for providing the spreadsheets of cancer registrations and incidence and Dr Subodh Gupta for doing the statistical analysis.

Statements on funding and competing interests

Funding None identified.

Competing interests None identified.

\section{References}

1 Bray F, Loos AH, McCarron P, Weiderpass E, Arbyn M, Møller $\mathrm{H}$, et al. Trends in cervical squamous cell carcinoma incidence in 13 European countries: changing risk and effects of screening. Cancer Epidemiol Biomarkers Prev 2005; 14: 677-686.

2 Peto J, Gilham C, Fletcher O, Matthews FE. The cervical cancer epidemic that screening has prevented in the UK. Lancet 2004; 364: 249-256.

3 National Health Service Cervical Screening Programme (NHSCSP). http://www.cancerscreening.nhs.uk/cervical/index. html\#eligible [Accessed 17 November 2007].

4 Sasieni P, Adams J, Cuzick J. Benefit of cervical screening at different ages: evidence from the UK audit of screening histories. Br J Cancer 2003; 89: 88-93.

5 International Agency for Research on Cancer (IARC). Uses of screening for cervical cancer (Chapter 3); Recommendations for public health implementation and further research (Chapter 8). Cervix Cancer Screening (IARC Handbooks of Cancer Prevention, Vol. 10). Lyon, France: IARC Press, 2005. 2005; 117-162; 239-241.

6 Rieck GC, Tristram A, Hauke A, Fielder H, Fiander AN. Cervical screening in 20-24-year olds. J Med Screen 2006; 13: 64-71.

7 Herbert A, Smith JHF. Women under 25 should be offered screening [Letter]. BMJ 2007; 334: 273 .

8 Nair MS, Bhandari HM, Nordin AJ. Cervical cancer in women aged less than 25: East Kent experience. J Obstet Gynaecol 2007; 27: 706-708.

9 The Information Centre (part of the Government Statistical Service). Cervical Screening Programme England 2005-06. http://www.cancerscreening.nhs.uk/cervical/cervical-statisticsbulletin-2005-06.pdf [Accessed 15 November 2007].

10 Sigurdsson K, Sigvaldason $\mathrm{H}$. Is it rational to start populationbased cervical cancer screening at or soon after age 20 ? Analysis of time trends in preinvasive and invasive disease. Eur J Cancer 2007; 43: 769-774.

11 National Health Service Cervical Screening Programme (NHSCSP). Audit of Invasive Cervical Cancers. http://www.cancerscreening.nhs.uk/cervical/publications/nhscs p28.pdf [Accessed 15 November 2007].

12 Office for National Statistics. http://www.statistics.gov.uk/ StatBase/Product.asp?vlnk=8843 [Accessed 15 November 2007].

13 Arbyn M, Dillner J, Schenck U, Nieminen P, Weiderpass E, Baldauf J-J, et al. Methods for screening and diagnosis (Chapter 3). In: Arbyn M, Anttila A, Jordan J, Ronco G, Schenck U, Segnan N, et al. (eds), European Guidelines for Quality Assurance in Cervical Cancer Screening (2nd edn). Luxembourg, Luxembourg: Office for Official Publications of the European Communities, 2007.

14 Wellings K, Nanchahal K, Macdowall W, McManus S, Erens B, Mercer $\mathrm{CH}$, et al. Sexual behaviour in Britain: early heterosexual experience. Lancet 2001; 358: 1843-1850.

15 The Information Centre (part of the Government Statistical Service). Cervical Screening Programme England 2006-07. http://www.ic.nhs.uk/webfiles/publications/cervicscrneng2007/ Cervical\%20screening\%2006-07.pdf [Accessed 15 November 2007].

16 Tidbury P, Singer A, Jenkins D. CIN3: the role of lesion size in invasion. B J Obstet Gynaecol 1992; 99: 583-586.

17 Lawton FG. Microinvasive cancer of the cervix (Chapter 13). In:
Leusley DM, Shafi MI, Jordan JA (eds), Handbook of Colposcopy. London, UK: Arnold, 2002; 114-118.

18 Eaton L, Fowler JM. The management of early invasion and adenocarcinoma-in-situ (Chapter 18). In: Prendiville W, Rittor J, Tatti S, Twiggs L (eds), Colposcopy Management Options. London, UK: W B Saunders, 2003; 143-147.

19 Mitchell MF, Tortolero-Luna G, Wright T, Sarkar A, RichardsKortum R, Hong WK. Cervical human papillomavirus infection and intraepithelial neoplasia: a review. I Natl Cancer Inst Monogr 1996; 21: 17-25.

20 Boyes DA, Morrison B, Knox EG, Draper GJ, Miller AB. A cohort study of cervical cancer screening in British Columbia. Clin Invest Med 1982; 5: 1-29.

21 Mclndoe WA, McLean MR, Jones RW, Mullins PR. The invasive potential of carcinoma in situ of the cervix. Obstet Gynecol 1984; 64: 451-58.

22 Cartwright SR. The Report of the Committee of Inquiry into Allegations Concerning the Treatment of Cervical Cancer at National Women's Hospital and Into Other Related Matters. Auckland, New Zealand: Government Printing Office, 1988.

23 Chamberlain J. Reasons that some screening programmes fail to control cervical cancer. In: Hakama M, Miller AB, Day NE (eds), Screening for Cancer of the Uterine Cervix (IARC Scientific Publications No. 76). Lyons, France: International Agency for Research on Cancer, 1986; 161-168.

24 Janerich DT, Hadjimichael O, Schwartz PE, Lowell DM, Meigs $\mathrm{JW}$, Merino MJ, et al. The screening histories of women with invasive cervical cancer, Connecticut. Am J Public Health 1995; 85: 791-794.

25 DeMay RM. Cytopathology of false negatives preceding cervical carcinoma. Am J Obstet Gynecol 1996; 175: 1110-1113.

26 Sykes PH, Reddy J, Peddie DJ. Does regular cervical screening protect women from microinvasive squamous cell carcinoma of the cervix? A retrospective case-control study. Br J Obstet Gynaecol 2005; 112: 807-809.

27 Herbert A, Smith JAE. Cervical intraepithelial neoplasia grade III (CIN III) and invasive cervical carcinoma: the yawning gap and the treatment of risk. Cytopathology 1999; 10: 161-170.

28 Kitchener HC, Castle PE, Cox JT. Chapter 7: Achievements and limitations of cervical cytology screening. Vaccine 2006; 24(Suppl. 3): S63-S70.

29 Herbert A, Singh N, Smith JAE. Adenocarcinoma of the uterine cervix compared with squamous cell carcinoma: a 12-year study in Southampton and South-west Hampshire. Cytopathology 2001; 12: 26-36.

30 Pretorius R, Semrad N, Watring W, Fotheringham N Presentation of cervical cancer. Gynecol Oncol 1991; 42: 48-53.

31 Sigurdsson K, Hrafnkelsson J, Geirsson G, Gudmundsson J, Salvarsdottir A. Screening as a prognostic factor in cervical cancer: analysis of survival and prognostic factors based on Icelandic population data 1964-1988. Gynecol Obstet 1991; 43: 64-70.

32 Abu-Rustum NR, Sonoda Y, Black D, Levine DA, Chi DS, Barakat RR. Fertility-sparing radical abdominal trachelectomy for cervical carcinoma: technique and review of the literature. Gynecol Oncol 2006; 103: 807-813.

33 Vizcaino AP, Moreno V, Bosch FX, Munoz N, Barros-Dios XM, Parkin DM. International trends in the incidence of cervical cancer 1. Adenocarcinoma and adenosquamous carcinoma. Int J Cancer 1998; 75: 536-545.

34 Kyrgiou M, Koliopoulos G, Martin-Hirsch P, Arbyn M, Prendiville W, Paraskevaidis W. Obstetric outcomes after conservative treatment for intraepithelial or early invasive cervical lesions: systematic review and meta-analysis. Lancet 2006; 367: 489-498.

35 Bruinsma F, Lumley J, Tan J, Quinn M. Precancerous changes in the cervix and risk of pre-term birth. Br J Obstet Gynaecol 2007; 114: 70-80.

36 Sadler L, Saftlas A, Wang W, Exeter M, Whittaker J, McCowan $\mathrm{L}$. Treatment of cervical intraepithelial neoplasia and risk of preterm delivery. JAMA 2004; 291: 2100-2106.

37 Szarewski A, Jarvis MJ, Sasieni $P$, Anderson M, Edwards $R$ Steele SJ, et al. The effect of smoking cessation on cervical lesion size. Lancet 1996; 347: 941-943.

38 Piyathilake CJ, Henao OL, Macaluso M, Cornwell PE, Meleth $\mathrm{S}$, Heimburger DC, et al. Folate is associated with the natural history of high-risk human papillomaviruses. Cancer Res 2004; 64: 8788-8793.

39 Hogewoning CJA, Bleeker MCG, van den Brule AJC, Voorhorst FJ, Snijders PJF, Berkhof J, et al. Condom use promotes regression of cervical intraepithelial neoplasia and clearance of human papillomavirus: a randomized clinical trial. Int J Cancer 2003; 107: 811-816. 